COMMENT. CBZ-epoxide serum levels should be measured in carbamazepine-treated patients with unexplained seizure exacerbation or toxicity. Risk factors for CBZ-epoxide induced status and toxicity include high dose $\mathrm{CBZ}$, combination therapies, and patients with mental retardation who often require polytherapy. Valproate, primidone, and felbamate combined with CBZ may increase levels of CBZ-epoxide by altering metabolic conversion or breakdown. In contrast, phenytoin promotes the conversion of the epoxide into an inactive form and reduces risk of CBZ-related toxicity and seizure exacerbation.

Gabapentin, an AED having no drug interactions, should reduce the risk of these complications when polytherapy is considered essential.

\title{
PROPOFOL ANESTHESIA-INDUCED SEIZURES
}

A case of a healthy young man who developed seizures and generalized paroxysmal fast activity in the EEG following use of propofol for anesthesia in minor surgery is reported from the Department of Neurology, University of South Alabama, Mobile, AL. Myoclonic jerking and obtundation developed shortly after anesthesia and a generalized seizure associated with EEG paroxysmal fast activity and controlled with divalproex occurred the following day. (Nowack WJ, Jordan R. Propofol, seizures and generalized paroxysmal fast activity in the EEG. Clin Electroencephalogr July 1994;25:110-114). (Reprints: William J Nowack MD, Dept of Neurology, Univ of South Alabama, Mobile, AL 36617).

COMMENT. Propofol anesthesia has been associated with seizures, myoclonic jerking and opisthotonic posturing. Spikes, polyspikes and spike wave complexes in the EEG have also been reported. The fast activity in the EEG in the above case was considered to be epileptiform, resolving after short-term anticonvulsant therapy.

\section{SEIZURE DISORDERS}

\section{CORTICAL HYPOMETABOLISM IN WEST'S SYNDROME}

The association between changes on serial PET studies and the clinical course of 12 patients with newly diagnosed West's syndrome is reported from the Departments of Pediatrics and Radiology, Nagoya University School of Medicine, Nagoya, Japan. PET with FDG revealed diffuse or focal cortical hypometabolism in 11 patients, whereas MRI showed abnormalities in only 5 . In 7 patients with normal findings on a second PET, spasms ceased after treatment, whereas in 5 with persistent abnormalities on PET, spasms persisted or recurred or partial seizures developed. Patients with normal MRI and normal second PET studies had normal psychomotor development. (Maeda N, Watanabe K, Negoro $T$ et al. Evolutional changes of cortical hypometabolism in West's syndrome. Lancet June 25, 1994;343:1620-23). (Dr Norihide Maeda, Dept of Pediatrics, Nagoya Univ Sch of Medicine, 65 Turuma-cho, Showa-ku, Nagoya 466, Japan).

COMMENT. PET has been found of value in the preoperative evaluation of patients with West's syndrome (Chugani HT et al. Ann Neurol

1990;27:406-13). It may also be used in the assessment of prognosis. See also Ped Neur Briefs March 1992 and Progress in Pediatric Neurology II, Chicago, PNB Publ, 1994, for further reference to PET and infantile spasms. 